\title{
Enantioselective Organocatalytic Allylic Amination
}

Thomas B. Poulsen, Carlos Alemparte, and Karl Anker Jørgensen*

The Danish National Research Foundation: Center for Catalysis

Department of Chemistry, Aarhus University, DK-8000 Aarhus C, Denmark

e-mail:kaj@chem.au.dk

\section{Supporting Information}

\section{Contents}

Catalyst structures

General methods

Materials

General procedure for the allylic amination

Characterization data for the amination products

S4

Procedures and characterization data for derivatives of the amination products

Determination of the absolute configuration

References 


\section{Catalyst Structures}

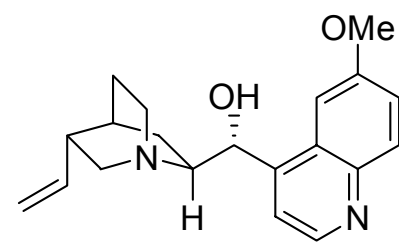

quinine<smiles>CCCN1CC2CCC1N2[C@H](O[14CH3])c1ccnc2ccc(OC)cc12</smiles>
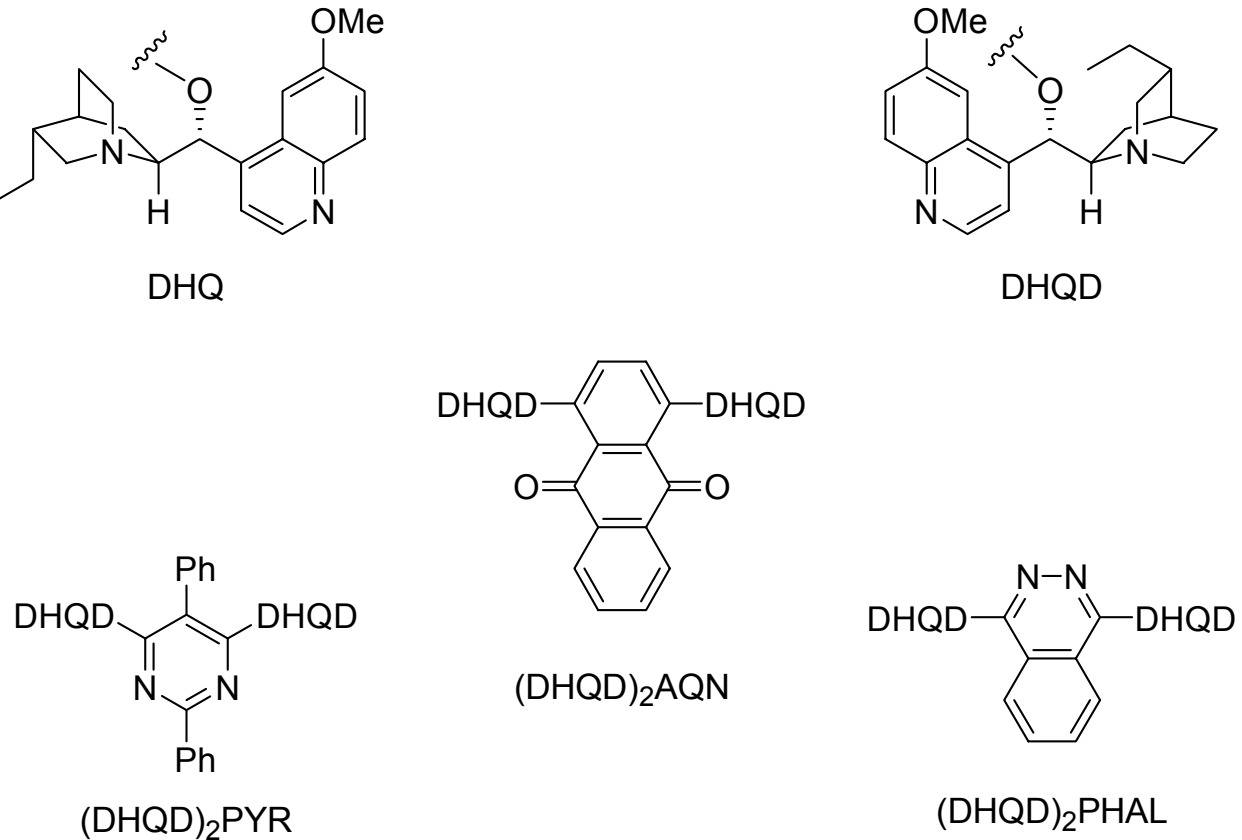
General Methods. NMR spectra were acquired on a Varian AS 400 spectrometer, running at 400 and $100 \mathrm{MHz}$ for ${ }^{1} \mathrm{H}$ and ${ }^{13} \mathrm{C}$, respectively. Chemical shifts $(\delta)$ are reported in ppm relative to residual solvent signals. All spectra were recorded at elevated temperatures $\left(60{ }^{\circ} \mathrm{C}\right)$ in order to minimize the effect of rotameric isomers. ${ }^{13} \mathrm{C}$ NMR spectra were acquired on a broad band decoupled mode. Mass spectra were recorded on a micromass LCT spectrometer using electrospray $\left(\mathrm{ES}^{+}\right)$ionization techniques. Flash column chromatography (FC) was carried out using the FlashMaster II from Jones Chromatography with columns containing silica gel. Analytical thin layer chromatography (TLC) was performed using pre-coated aluminium-backed plates (Merck Kieselgel 60 F254) and visualized by ultraviolet irradiation or $\mathrm{KMnO}_{4}$ dip. Optical rotations were measured on a Perkin-Elmer 241 polarimeter. The enantiomeric excess (ee) of the products was determined by chiral stationary phase HPLC (Daicel Chiralpak AS/AD or Daicel Chiralcel OD columns).

Materials. Analytical grade solvents were used as received. For flash chromatography (FC) silica gel was purchased from Iatron Laboratories Inc. (Iatrobeads 6RS-8060) or from Fluka (Silica gel 60, 230-400 mesh). Dialkyl azodicarboxylates and catalysts (2) are all commercially available and were used as received. Substrates 1a-k,o were prepared by Knoevenagel condensation of the appropriate $\alpha$-cyanoacetate and aldehyde following a literature procedure. ${ }^{1}$ Substrate 11 was synthesized by condensing malonitrile with butyraldehyde. ${ }^{2}$ Substrates $\mathbf{1} \mathbf{m}, \mathbf{n}$ were prepared from malononitrile and the corresponding ketones. ${ }^{3}$

General Procedure for allylic amination of alkylidene cyanoacetates: To a test tube equipped with a magnetic stirring bar were added dichloromethane $(1.0 \mathrm{~mL})$, di-tert-butyl azodicarboxylate $(0.24 \mathrm{mmol}, 55.4 \mathrm{mg})$, and the alkylidene cyanoacetate $(0.2 \mathrm{mmol})$. The test tube was fitted with a rubber septum, stirred at ambient temperature to dissolve the solids, and then cooled to $-78{ }^{\circ} \mathrm{C}$. (DHQ) ${ }_{2} \mathrm{PYR}(10 \mathrm{~mol} \%, 0.02 \mathrm{mmol}, 17.6 \mathrm{mg}$ ) was added as a solid to the cooled mixture, which was then placed at $-24^{\circ} \mathrm{C}$ for $41-47 \mathrm{~h}$. The mixture was then cooled to $-78{ }^{\circ} \mathrm{C}$ and loaded directly onto a chromatographic column 
containing Iatrobeads. The pure product was isolated by FC. The enantiomeric excess of the products was determined by HPLC using a chiral stationary phase.

\section{(R)-(E)-4-[N, $N^{\prime}$-Bis(tert-butoxycarbonyl)-hydrazino]-2-cyanohex-2-enoic acid benzyl ester (2a):}

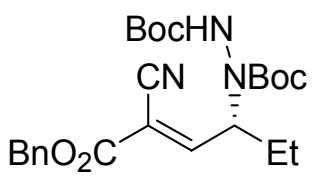

The title compound was obtained according to the general procedure, but using (DHQD) ${ }_{2} \mathrm{PYR}$ as the catalyst, after FC in $\mathrm{Et}_{2} \mathrm{O} / n$-hexane as a viscous colorless oil (83\% yield). ${ }^{1} \mathrm{H}$ NMR $\left(\mathrm{CDCl}_{3}, 60^{\circ} \mathrm{C}\right) \delta 7.67(\mathrm{~d}, J 9.1 \mathrm{~Hz}, 1 \mathrm{H}), 7.43-7.31(\mathrm{~m}, 5 \mathrm{H}), 6.11$ (br s, 1H), 5.30 (s, 2H), 4.94 (q, J $6.5 \mathrm{~Hz}, 1 \mathrm{H}), 1.88$ (m, 1H), 1.66 $(\mathrm{m}, 1 \mathrm{H}), 1.48(\mathrm{~s}, 9 \mathrm{H}), 1.46(\mathrm{~s}, 9 \mathrm{H}), 0.99(\mathrm{t}, J 7.4 \mathrm{~Hz}, 3 \mathrm{H}) .{ }^{13} \mathrm{C} \mathrm{NMR}\left(\mathrm{CDCl}_{3}, 60{ }^{\circ} \mathrm{C}\right) \delta$ $160.9,160.3,155.9,154.3,134.9,128.6$ (2C), 128.5 (2C), 128.2, 113.0, 109.3, 82.5, 81.9, 68.0, 60.3, 28.2 (3C), 28.0 (3C), 24.8, 10.4. HRMS calc.: $\mathrm{C}_{24} \mathrm{H}_{33} \mathrm{~N}_{3} \mathrm{NaO}_{6}$ 482.2267; found: 482.2270. The ee was determined by HPLC using a Chiralpak AD column [hexane $/ \mathrm{iPrOH}(75: 25)$ ]; flow rate $1.0 \mathrm{~mL} / \mathrm{min} ; \tau_{\text {major }}=18.2 \mathrm{~min}, \tau_{\mathrm{minor}}=11.8 \mathrm{~min}(94 \%$ ee).

(S)-(E)-4-[N, $N^{\prime}$-Bis(tert-butoxycarbonyl)-hydrazino]-2-cyanohex-2-enoic acid allyl ester (2b):

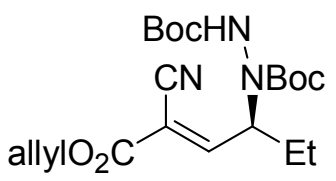

The title compound was obtained according to the general procedure after $\mathrm{FC}$ in $\mathrm{Et}_{2} \mathrm{O} / n$-hexane as a viscous colorless oil (90\% yield). ${ }^{1} \mathrm{H} \mathrm{NMR}\left(\mathrm{CDCl}_{3}, 60{ }^{\circ} \mathrm{C}\right) \delta 7.66(\mathrm{~d}, J 9.1 \mathrm{~Hz}, 1 \mathrm{H})$, 6.12 (br s, 1H), 5.96 (ddt, $J 16.2,10.8,5.8 \mathrm{~Hz}, 1 \mathrm{H}), 5.40$ (dd, $J$ 17.2, 1.4 Hz, 1H), 5.30 (dd, $J 10.6,1.1 \mathrm{~Hz}, 1 \mathrm{H}), 4.95$ (q, $J 8.2$ $\mathrm{Hz}, 1 \mathrm{H}), 4.75$ (d, J $5.7 \mathrm{~Hz}, 2 \mathrm{H}), 1.88$ (m, 1H), 1.67 (m, 1H), 1.49 (s, 9H), 1.49 (s, 9H), $1.00(\mathrm{t}, J 7.4 \mathrm{~Hz}, 3 \mathrm{H}) .{ }^{13} \mathrm{C} \mathrm{NMR}\left(\mathrm{CDCl}_{3}, 60{ }^{\circ} \mathrm{C}\right) \delta 160.7,160.2,156.0,154.3,131.0$, 119.2, 113.0, 109.2, 82.5, 81.9, 66.8, 60.3, 28.2 (3C), 28.1 (3C), 24.8, 10.4. HRMS calc.: $\mathrm{C}_{20} \mathrm{H}_{31} \mathrm{~N}_{3} \mathrm{NaO}_{6}$ 432.2111; found: 432.2104. The ee was determined by HPLC using a Chiralpak AD column [hexane $/ \mathrm{PrOH}(75: 25)]$; flow rate $1.0 \mathrm{~mL} / \mathrm{min} ; \tau_{\text {major }}=7.0 \mathrm{~min}$, $\tau_{\text {minor }}=11.4 \min (97 \%$ ee $)$. 
$(S)-(E)-4-\left[N, N^{\prime}\right.$-Bis(tert-butoxycarbonyl)-hydrazino]-2-cyanohex-2-enoic acid methyl ester (2c):<smiles>CCC(C=C(C)C(C)=O)[N+](=O)[O-]</smiles>

The title compound was obtained according to the general procedure after $\mathrm{FC}$ in $\mathrm{Et}_{2} \mathrm{O} / n$-hexane as a viscous colorless oil (84\% yield). ${ }^{1} \mathrm{H} \mathrm{NMR}\left(\mathrm{CDCl}_{3}, 60{ }^{\circ} \mathrm{C}\right) \delta 7.65(\mathrm{~d}, J 9.2 \mathrm{~Hz}, 1 \mathrm{H})$, 6.12 (br s, 1H), $4.93(\mathrm{q}, J 8.1 \mathrm{~Hz}, 1 \mathrm{H}), 3.87(\mathrm{~s}, 3 \mathrm{H}), 1.87(\mathrm{~m}, 1 \mathrm{H})$, $1.66(\mathrm{~m}, 1 \mathrm{H}), 1.49(\mathrm{~s}, 18 \mathrm{H}), 0.99(\mathrm{t}, J 9.2 \mathrm{~Hz}, 3 \mathrm{H}) .{ }^{13} \mathrm{C}$ NMR $\left(\mathrm{CDCl}_{3}, 60{ }^{\circ} \mathrm{C}\right)^{5} \delta 161.5,160.2,156.0,154.3,113.1,109.1,82.5,82.0,60.3,53.1,53.0$, 28.2 (3C), 28.1 (3C), 24.8, 10.4. HRMS calc.: $\mathrm{C}_{18} \mathrm{H}_{29} \mathrm{~N}_{3} \mathrm{NaO}_{6}$ 406.1954; Found: 406.1942. The ee was determined by HPLC using a Chiralpak AD column [hexane $/ \mathrm{iPrOH}(75: 25)]$; flow rate $1.0 \mathrm{~mL} / \mathrm{min} ; \tau_{\text {major }}=6.4 \mathrm{~min}, \tau_{\text {minor }}=14.3 \mathrm{~min}(98 \%$ ee).

$(R)-(E)-4-\left[N, N^{\prime}\right.$-Bis(tert-butoxycarbonyl)-hydrazino]-2-cyanohex-2-enoic acid ethyl ester (2d):

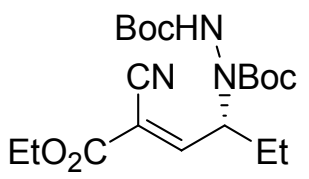

The title compound was obtained according to the general procedure, but using (DHQD) $)_{2} \mathrm{PYR}$ as the catalyst, after $\mathrm{FC}$ in $\mathrm{Et}_{2} \mathrm{O} / n$-hexane as a viscous colorless oil $\left(71 \%\right.$ yield). ${ }^{1} \mathrm{H}$ NMR $\left(\mathrm{CDCl}_{3}, 60{ }^{\circ} \mathrm{C}\right) \delta 7.63(\mathrm{~d}, J 9.2 \mathrm{~Hz}, 1 \mathrm{H}), 6.11(\mathrm{br}, \mathrm{s}, 1 \mathrm{H}), 4.94(\mathrm{q}$, $J 8.1 \mathrm{~Hz}, 1 \mathrm{H}), 4.33(\mathrm{q}, J 7.1 \mathrm{~Hz}, 2 \mathrm{H}), 1.88(\mathrm{~m}, 1 \mathrm{H}), 1.66(\mathrm{~m}, 1 \mathrm{H})$, 1.49 (s, 9H), 1.49 (s, 9H), 1.37 (t, $J 7.1 \mathrm{~Hz}, 3 \mathrm{H}), 0.99$ (t, $J 7.4 \mathrm{~Hz}, 3 \mathrm{H}) .{ }^{13} \mathrm{C} \mathrm{NMR}\left(\mathrm{CDCl}_{3}\right.$, $\left.60{ }^{\circ} \mathrm{C}\right) \delta 161.0,159.7,156.0,154.3,113.1,109.5,82.5,81.9,62.5,60.3,28.2(3 \mathrm{C}), 28.1$ (3C), 24.8, 14.0, 10.4. HRMS calc.: $\mathrm{C}_{19} \mathrm{H}_{31} \mathrm{~N}_{3} \mathrm{NaO}_{6}$ 420.2111; found: 420.2127. The ee was determined by HPLC using a Chiralpak AD column [hexane/iPrOH (75:25)]; flow rate $1.0 \mathrm{~mL} / \mathrm{min} ; \tau_{\text {major }}=12.2 \mathrm{~min}, \tau_{\text {minor }}=6.3 \mathrm{~min}(90 \%$ ee $)$. 
$(R)-(E)-4-\left[N, N^{\prime}\right.$-Bis(tert-butoxycarbonyl)-hydrazino]-2-cyanohex-2-enoic $\quad$ acid $\quad i$ propyl ester (2e):

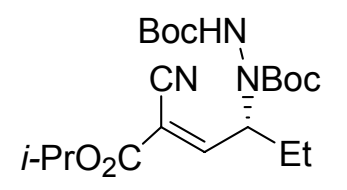

The title compound was obtained according to the general procedure, but using (DHQD) $)_{2} \mathrm{PYR}$ as the catalyst, after FC in $\mathrm{Et}_{2} \mathrm{O} / n$-hexane as a viscous colorless oil (62\% yield). ${ }^{1} \mathrm{H}$ NMR $\left(\mathrm{CDCl}_{3}, 60{ }^{\circ} \mathrm{C}\right) \delta 7.60(\mathrm{~d}, J 9.1 \mathrm{~Hz}, 1 \mathrm{H}), 6.11(\mathrm{br} \mathrm{s}, 1 \mathrm{H}), 5.14$ (septet, $J 6.2 \mathrm{~Hz}, 1 \mathrm{H}), 4.95$ (q, $J 8.3 \mathrm{~Hz}, 1 \mathrm{H}), 1.88(\mathrm{~m}, 1 \mathrm{H}), 1.66$

(m, 1H), 1.49 (s, 9H), 1.49 (s, 9H), $1.34(\mathrm{~d}, J 6.3 \mathrm{~Hz}, 6 \mathrm{H}), 0.99$ (t, $J 7.4 \mathrm{~Hz}, 3 \mathrm{H}) .{ }^{13} \mathrm{C}$ $\operatorname{NMR}\left(\mathrm{CDCl}_{3}, 60{ }^{\circ} \mathrm{C}\right)^{5} \delta 160.5,159.4,156.0,154.3,113.2,110.0,82.5,81.9,70.8,70.7$, 60.3, 28.2 (3C), 28.1 (3C), 24.9, 21.6 (2C), 10.4. HRMS calc.: $\mathrm{C}_{20} \mathrm{H}_{33} \mathrm{~N}_{3} \mathrm{NaO}_{6} 434.2267$; found: 420.2250 . The ee was determined by HPLC using a Chiralpak AD column [hexane $/ \mathrm{PrOH}(75: 25)$ ]; flow rate $1.0 \mathrm{~mL} / \mathrm{min} ; \tau_{\text {major }}=9.6 \mathrm{~min}, \tau_{\text {minor }}=5.8 \mathrm{~min}(93 \%$ ee).

$(R)-(E)-4-\left[N, N^{\prime}\right.$-Bis(tert-butoxycarbonyl)-hydrazino]-2-cyanohex-2-enoic acid $t$-butyl ester (2f):

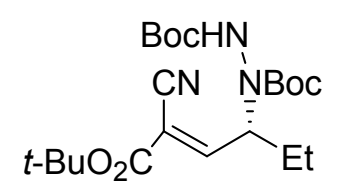

The title compound was obtained according to the general procedure, but using (DHQD) 2 PYR as the catalyst, after FC in $\mathrm{Et}_{2} \mathrm{O} / n$-hexane as a viscous colorless oil (21\% yield). ${ }^{1} \mathrm{H}$ NMR $\left(\mathrm{CDCl}_{3}, 60{ }^{\circ} \mathrm{C}\right) \delta 7.53(\mathrm{~d}, J 9.1 \mathrm{~Hz}, 1 \mathrm{H}), 6.12(\mathrm{br} \mathrm{s}, 1 \mathrm{H}), 4.93(\mathrm{q}$, $J 7.8 \mathrm{~Hz}, 1 \mathrm{H}), 1.86(\mathrm{~m}, 1 \mathrm{H}), 1.64(\mathrm{~m}, 1 \mathrm{H}), 1.54(\mathrm{~s}, 9 \mathrm{H}), 1.49(\mathrm{~s}$,

9H), 1.48 (s, 9H), 0.99 (t, J 7.4 Hz, 3H). ${ }^{13} \mathrm{C} \mathrm{NMR}\left(\mathrm{CDCl}_{3}, 60{ }^{\circ} \mathrm{C}\right) \delta 159.9,158.6,156.0$, 154.4, 113.5, 111.1, 83.8, 82.5, 82.0, 60.2, 28.2 (3C), 28.1 (3C), 28.0 (3C), 24.9, 10.6. HRMS calc.: $\mathrm{C}_{21} \mathrm{H}_{35} \mathrm{~N}_{3} \mathrm{NaO}_{6}$ 448.2424; found: 448.2427. The ee was determined by HPLC using a Chiralpak AD column [hexane $/ \mathrm{PrOH}$ (75:25)]; flow rate $1.0 \mathrm{~mL} / \mathrm{min}$; $\tau_{\text {major }}=8.2 \mathrm{~min}, \tau_{\text {minor }}=5.5 \mathrm{~min}(91 \%$ ee $)$. 


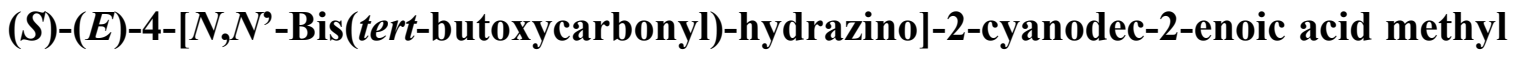
ester (2g):<smiles>COC(=O)C(C)=C[C@H](NC(=O)OCc1ccccc1)C(=O)OCc1ccccc1</smiles>

The title compound was obtained according to the general procedure after $\mathrm{FC}$ in $\mathrm{Et}_{2} \mathrm{O} / n$-hexane as a viscous colorless oil (90\% yield). ${ }^{1} \mathrm{H}$ NMR $\left(\mathrm{CDCl}_{3}, 60{ }^{\circ} \mathrm{C}\right) \delta 7.65(\mathrm{~d}, J 9.2 \mathrm{~Hz}, 1 \mathrm{H})$, $6.10(\mathrm{br} \mathrm{s}, 1 \mathrm{H}), 5.02$ (q, $J 8.2 \mathrm{~Hz}, 1 \mathrm{H}), 3.87$ (s, 3H), $1.84(\mathrm{~m}$, $1 \mathrm{H}), 1.58(\mathrm{~m}, 1 \mathrm{H}), 1.49(\mathrm{~s}, 18 \mathrm{H}), 1.41-1.25(\mathrm{~m}, 8 \mathrm{H}), 0.90(\mathrm{t}, J$ $6.7 \mathrm{~Hz}, 3 \mathrm{H}) .{ }^{13} \mathrm{C} \mathrm{NMR}\left(\mathrm{CDCl}_{3}, 60{ }^{\circ} \mathrm{C}\right)^{5} \delta 161.5,160.4,156.0,154.2,113.0,108.8,82.5$, 82.0, 58.7, 53.1, 53.0, 31.5, 31.4, 28.8, 28.2 (3C), 28.1 (3C), 25.7, 22.4, 13.8. HRMS calc.: $\mathrm{C}_{22} \mathrm{H}_{37} \mathrm{~N}_{3} \mathrm{NaO}_{6} 462.2580$; found: 462.2590 . $[\alpha]_{\mathrm{D}}{ }^{20}+70\left(c=1.0, \mathrm{CHCl}_{3}, 99 \%\right.$ ee). The ee was determined by HPLC using a Chiralpak AD column [hexane/iPrOH (75:25)]; flow rate $1.0 \mathrm{~mL} / \mathrm{min} ; \tau_{\text {major }}=5.2 \mathrm{~min}, \tau_{\operatorname{minor}}=9.9 \mathrm{~min}(99 \%$ ee $)$.

\section{(S)-(E)-4-[N, $N^{\prime}$-Bis(tert-butoxycarbonyl)-hydrazino]-2-cyano-5-methylhex-2-enoic} acid methyl ester (2h):

The title compound was obtained according to the general $\mathrm{MeO}_{2} \overbrace{i-\mathrm{Pr}}^{\mathrm{BocHN}}$ procedure, but at a temperature of $4{ }^{\circ} \mathrm{C}$, after $\mathrm{FC}$ in $\mathrm{Et}_{2} \mathrm{O} / n$ hexane as a viscous colorless oil (87\% yield). ${ }^{1} \mathrm{H} \mathrm{NMR}\left(\mathrm{CDCl}_{3}\right.$, $\left.60{ }^{\circ} \mathrm{C}\right) \delta 7.57(\mathrm{~d}, J 10.4,1 \mathrm{H}), 6.01(\mathrm{br} \mathrm{s}, 1 \mathrm{H}), 4.71(\mathrm{t}, J 10.3 \mathrm{~Hz}$, 1H), $3.88(\mathrm{~s}, 3 \mathrm{H}), 2.08(\mathrm{~m}, 1 \mathrm{H}), 1.50(\mathrm{~s}, 9 \mathrm{H}), 1.48(\mathrm{~s}, 9 \mathrm{H}), 1.05$ $(\mathrm{d}, J 6.6 \mathrm{~Hz}, 3 \mathrm{H}), 0.93(\mathrm{~d}, J 6.8 \mathrm{~Hz}, 3 \mathrm{H}) .{ }^{13} \mathrm{C} \mathrm{NMR}\left(\mathrm{CDCl}_{3}, 60{ }^{\circ} \mathrm{C}\right)^{5} \delta 161.5,158.6$, 156.0, 154.3, 113.3, 110.2, 82.6, 82.0, 64.7, 53.1, 53.0, 30.0, 28.2 (3C), 28.0 (3C), 19.7, 18.7. HRMS calc.: $\mathrm{C}_{19} \mathrm{H}_{31} \mathrm{~N}_{3} \mathrm{NaO}_{6} 420.2111$; found: 420.2110 . $[\alpha]_{\mathrm{D}}{ }^{20}+103(c=1.0$, $\mathrm{CHCl}_{3}, 96 \%$ ee). The ee was determined by HPLC using a Chiralpak AD column [hexane $/ \mathrm{iPrOH}(80: 20)]$; flow rate $1.0 \mathrm{~mL} / \mathrm{min} ; \tau_{\text {major }}=7.8 \mathrm{~min}, \tau_{\text {minor }}=18.7 \mathrm{~min}(90 \%$ ee). 


\section{$(S)-(E)-4-\left[N, N^{\prime}\right.$-Bis(tert-butoxycarbonyl)-hydrazino]-2-cyano-5-phenylpent-2-enoic}

acid methyl ester (2i):

The title compound was obtained according to the general $\mathrm{MeO}_{2} \mathrm{C}$ procedure after $\mathrm{FC}$ in $\mathrm{Et}_{2} \mathrm{O} / n$-hexane as a viscous colorless oil (89\% yield). ${ }^{1} \mathrm{H}$ NMR $\left(\mathrm{CDCl}_{3}, 60{ }^{\circ} \mathrm{C}\right) \delta 7.75(\mathrm{~d}, J 8.9 \mathrm{~Hz}, 1 \mathrm{H})$, $7.32(\mathrm{~m}, 2 \mathrm{H}), 7.23(\mathrm{~m}, 3 \mathrm{H}), 6.03$ (br s, 1H), $5.34(\mathrm{~m}, 1 \mathrm{H}), 3.86$ (s, 3H), $3.16(\mathrm{~m}, 1 \mathrm{H}), 2.96(\mathrm{~m}, 1 \mathrm{H}), 1.49(\mathrm{~s}, 9 \mathrm{H}), 1.41(\mathrm{~s}, 9 \mathrm{H})$. ${ }^{13} \mathrm{C}$ NMR $\left(\mathrm{CDCl}_{3}, 60{ }^{\circ} \mathrm{C}\right){ }^{5} \delta 161.3,159.4,156.0,153.9,135.9,129.1(2 \mathrm{C}), 128.8(2 \mathrm{C})$, 127.2, 112.9, 109.3, 82.6, 82.2, 60.5, 53.1, 53.0, 37.8, 28.1 (6C). HRMS calc.: $\mathrm{C}_{23} \mathrm{H}_{31} \mathrm{~N}_{3} \mathrm{NaO}_{6}$ 468.2111; found: 468.2103. $[\alpha]_{\mathrm{D}}{ }^{20}+101$ ( $c=1.0, \mathrm{CH}_{2} \mathrm{Cl}_{2}, 98 \%$ ee). The ee was determined by HPLC using a Chiralpak AD column [hexane/iPrOH (80:20)]; flow rate $1.0 \mathrm{~mL} / \mathrm{min} ; \tau_{\text {major }}=8.3 \mathrm{~min}, \tau_{\text {minor }}=21.1 \mathrm{~min}(98 \%$ ee $)$.

$(S)-(E)-4-\left[N, N^{\prime}-B i s(t e r t-b u t o x y c a r b o n y l)-h y d r a z i n o\right]-2-c y a n o o c t a-2,7-d i e n o i c \quad$ acid methyl ester $(2 \mathbf{j})$ :

The title compound was obtained according to the general $\mathrm{MeO}_{2} \mathrm{C}$ procedure after $\mathrm{FC}$ in $\mathrm{Et}_{2} \mathrm{O} / n$-hexane as a viscous colorless oil (85\% yield). ${ }^{1} \mathrm{H}$ NMR $\left(\mathrm{CDCl}_{3}, 60{ }^{\circ} \mathrm{C}\right) \delta 7.67(\mathrm{~d}, J 8.9 \mathrm{~Hz}, 1 \mathrm{H})$, 6.12 (br s, 1H), 5.77 (ddt, $J 17.1,10.1,7.0 \mathrm{~Hz}, 1 \mathrm{H}), 5.21-5.06$ $(\mathrm{m}, 3 \mathrm{H}), 3.87(\mathrm{~s}, 3 \mathrm{H}), 2.63(\mathrm{~m}, 1 \mathrm{H}), 2.42(\mathrm{~m}, 1 \mathrm{H}), 1.49(\mathrm{~s}, 9 \mathrm{H})$, 1.49 (s, 9H). ${ }^{13} \mathrm{C} \mathrm{NMR}\left(\mathrm{CDCl}_{3}, 60{ }^{\circ} \mathrm{C}\right)^{5} \delta 161.4,159.6,155.9,154.0,132.6,119.0,113.0$, 109.2, 82.6, 82.0, 58.5, 53.1, 53.0, 35.8, 28.1 (3C), 28.1 (3C). HRMS calc.: $\mathrm{C}_{19} \mathrm{H}_{29} \mathrm{~N}_{3} \mathrm{NaO}_{6}$ 418.1954; found: 418.1958. $[\alpha]_{\mathrm{D}}{ }^{20}+69\left(c=1.0, \mathrm{CHCl}_{3}, 96 \%\right.$ ee). The ee was determined by HPLC using a Chiralpak AD column [hexane/iPrOH (80:20)]; flow rate $1.0 \mathrm{~mL} / \mathrm{min} ; \tau_{\text {major }}=7.6 \mathrm{~min}, \tau_{\text {minor }}=19.4 \mathrm{~min}(96 \%$ ee $)$. 


\section{$(S)-(E)-4-\left[N, N^{\prime}\right.$-Bis(tert-butoxycarbonyl)-hydrazino]-7-(tert-butyldimethyl-}

silanyloxy)-2-cyanohept-2-enoic acid methyl ester (2k):

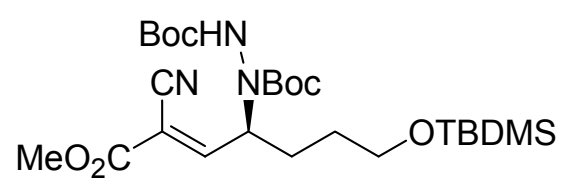

The title compound was obtained according to the general procedure after $\mathrm{FC}$ in $\mathrm{Et}_{2} \mathrm{O} / \mathrm{CH}_{2} \mathrm{Cl}_{2}$ as a viscous colorless oil ( $80 \%$ yield). ${ }^{1} \mathrm{H}$ NMR $\left(\mathrm{CDCl}_{3}\right.$, $\left.60{ }^{\circ} \mathrm{C}\right) \delta 7.66(\mathrm{~d}, J 9.1 \mathrm{~Hz}, 1 \mathrm{H}), 6.24($ br s, 1H) 5.04

(br m, 1H), 3.87 (s, 3H), 3.66 (t, J 6.4 Hz, 2H), 1.94 (m, 1H), 1.70-1.55 (m, 3H), 1.49 (br $\mathrm{s}, 18 \mathrm{H}), 0.91(\mathrm{~s}, 9 \mathrm{H}), 0.07(\mathrm{~s}, 6 \mathrm{H}) .{ }^{13} \mathrm{C} \mathrm{NMR}\left(\mathrm{CDCl}_{3}, 60{ }^{\circ} \mathrm{C}\right){ }^{5} \delta 161.5,160.3,155.9$, $154.2,113.0,108.9,82.5,81.9,62.3,58.6,53.1,53.0,29.2,28.2$ (3C), 28.1 (3C), 27.8, 26.0 (3C), 18.3, -5.3, -5.4. HRMS calc.: $\mathrm{C}_{25} \mathrm{H}_{45} \mathrm{~N}_{3} \mathrm{NaO}_{7} \mathrm{Si}$ 550.2924; found: 550.2928. $[\alpha]_{\mathrm{D}}^{20}+60\left(c=0.33, \mathrm{CHCl}_{3}, 97 \%\right.$ ee $)$. The ee was determined by HPLC using a Chiralpak AD column [hexane $/ \mathrm{PrOH}(90: 10)]$; flow rate $1.0 \mathrm{~mL} / \mathrm{min} ; \tau_{\text {major }}=7.7 \mathrm{~min}$, $\tau_{\text {minor }}=13.2 \min (97 \%$ ee $)$.

(S)-4-[N, $N^{\prime}$-Bis(tert-butoxycarbonyl)-hydrazino]-2-cyanohex-2-en nitrile (2I):

The title compound was obtained according to the general<smiles>CC[C@H](/C=C(\[NH3+])C#N)NNC(=O)O</smiles>
procedure after $\mathrm{FC}$ in $\mathrm{Et}_{2} \mathrm{O} / n$-hexane as a viscous colorless oil (65\% yield). ${ }^{1} \mathrm{H} \mathrm{NMR}\left(\mathrm{CDCl}_{3}, 60^{\circ} \mathrm{C}\right) \delta 7.44$ (br d, $\left.J 9.5 \mathrm{~Hz}, 1 \mathrm{H}\right)$, 6.16 (br s, 1H), 4.84 (q, J $8.0 \mathrm{~Hz}, 1 \mathrm{H}), 1.89$ (m, 1H), 1.67 (m, 1H), $1.50(\mathrm{~s}, 9 \mathrm{H}), 1.50(\mathrm{~s}, 9 \mathrm{H}), 1.00(\mathrm{t}, J 7.4 \mathrm{~Hz}, 3 \mathrm{H}) .{ }^{13} \mathrm{C} \mathrm{NMR}$ $\left(\mathrm{CDCl}_{3}, 60^{\circ} \mathrm{C}\right) \delta 166.5,156.2,154.1,111.9,110.3,88.6,83.2,82.4,60.9,28.2,28.1$, 24.5, 10.3. HRMS calc.: $\mathrm{C}_{17} \mathrm{H}_{26} \mathrm{~N}_{4} \mathrm{O}_{4}$ 373.1852; found: 373.1841. $[\alpha]_{\mathrm{D}}{ }^{20}+90(c=1.0$, $\mathrm{CHCl}_{3}, 91 \%$ ee). The ee was determined by HPLC using a Chiralpak AD column [hexane $/ \mathrm{PrOH}(75: 25)]$; flow rate $1.0 \mathrm{~mL} / \mathrm{min} ; \tau_{\text {major }}=4.0 \mathrm{~min}, \tau_{\text {minor }}=6.3 \mathrm{~min}(91 \%$ ee). 
(S)-4-[N, $N^{\prime}$-Bis(tert-butoxycarbonyl-hydrazino]-2-cyano-3-phenylpent-2-en nitrile (2m):

$\overbrace{\mathrm{Ph}}^{\mathrm{BocHN}}$

The title compound was obtained according to the general procedure after $\mathrm{FC}$ in $\mathrm{Et}_{2} \mathrm{O} / \mathrm{CH}_{2} \mathrm{Cl}_{2}$ as a viscous colorless oil (85\% yield). ${ }^{1} \mathrm{H}$ NMR $\left(\mathrm{CDCl}_{3}, 60{ }^{\circ} \mathrm{C}\right) \delta$ 7.69-7.47 (m, 5H), 6.41 (br s, 1H), 5.19 (m, 1H), 1.51 (s, 9H), 1.50 (s, 9H), 1.35 (br d, $J$ $6.2 \mathrm{~Hz}, 3 \mathrm{H}) .{ }^{13} \mathrm{C}$ NMR Due to severe rotameric broadening of many of the signals, the spectrum is attached below. HRMS calc.: $\mathrm{C}_{22} \mathrm{H}_{28} \mathrm{~N}_{4} \mathrm{NaO}_{4} 435.2008$; found: 435.2010 . $[\alpha]_{\mathrm{D}}{ }^{20}+250$ (c=0.50, $\mathrm{CHCl}_{3}, 86 \%$ ee). The ee was determined by HPLC using two Chiralpak AS columns coupled in series [hexane $/ \mathrm{iPrOH}(98: 2)$ ]; flow rate $1.0 \mathrm{~mL} / \mathrm{min} ; \tau_{\text {major }}=12.5 \mathrm{~min}, \tau_{\text {minor }}=10.8 \mathrm{~min}(86 \%$ ee).

\section{(S)-4-(N,N'-Bis(tert-butoxycarbonyl-hydrazino)-2-cyano-3,5-diphenylpent-2-en}

nitrile (2n):

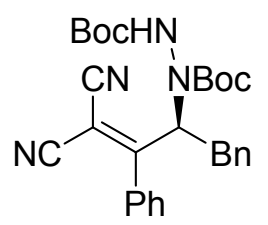

The title compound was obtained according to the general procedure after $\mathrm{FC}$ in $\mathrm{Et}_{2} \mathrm{O} / \mathrm{CH}_{2} \mathrm{Cl}_{2}$ as a colorless solid ( $\mathrm{mp}=$ $48-50{ }^{\circ} \mathrm{C}, 84 \%$ yield). ${ }^{1} \mathrm{H}$ NMR $\left(\mathrm{CDCl}_{3}, 60{ }^{\circ} \mathrm{C}\right) \delta$ 7.53-7.25 (m, $5 \mathrm{H}), 7.23-6.97$ (m, 5H), 6.43 (br s, 1H), 5.56 (br t, $J 7.3 \mathrm{~Hz}$, $1 \mathrm{H}), 3.36$ (dd, $J 13.8,6.7 \mathrm{~Hz}, 1 \mathrm{H}), 3.03(\mathrm{dd}, J 13.8,9.4 \mathrm{~Hz}, 1 \mathrm{H})$, $1.52(\mathrm{~s}, 9 \mathrm{H}), 1.50(\mathrm{~s}, 9 \mathrm{H}) \cdot{ }^{13} \mathrm{C}$ NMR Due to severe rotameric broadening of many of the signals, the spectrum is attached below. HRMS calc.: $\mathrm{C}_{28} \mathrm{H}_{32} \mathrm{~N}_{4} \mathrm{NaO}_{4} 511.2321$; found: 511.2320. $[\alpha]_{\mathrm{D}}^{20}+346\left(\mathrm{c}=0.49, \mathrm{CHCl}_{3}, 88 \%\right.$ ee). The ee was determined by HPLC using a Chiralpak AD column [hexane $/ \mathrm{PrOH}(90: 10)]$; flow rate $1.0 \mathrm{~mL} / \mathrm{min} ; \tau_{\text {major }}=9.1$ $\min , \tau_{\operatorname{minor}}=13.3 \min (88 \%$ ee $)$. 


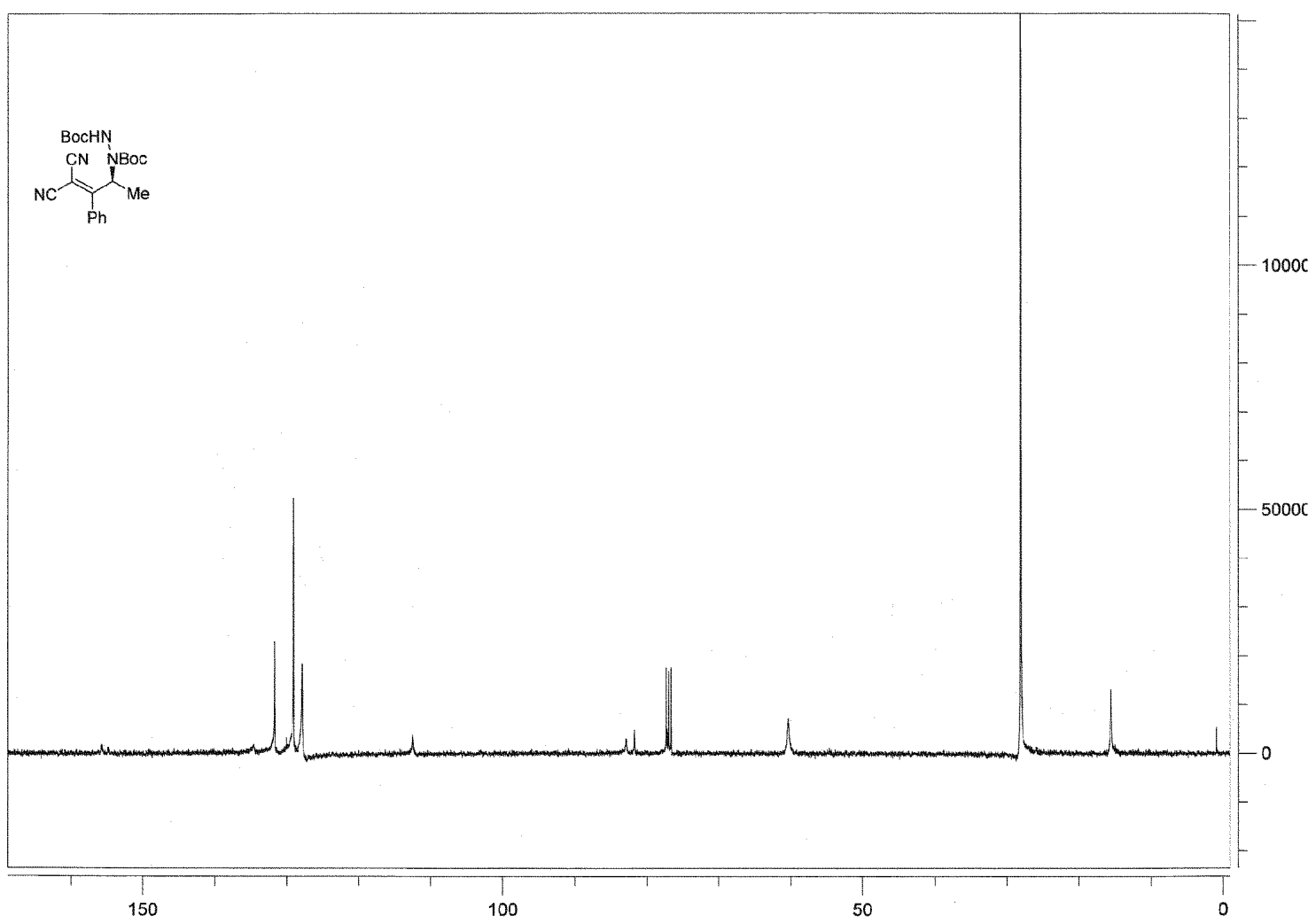

ppm (f1)

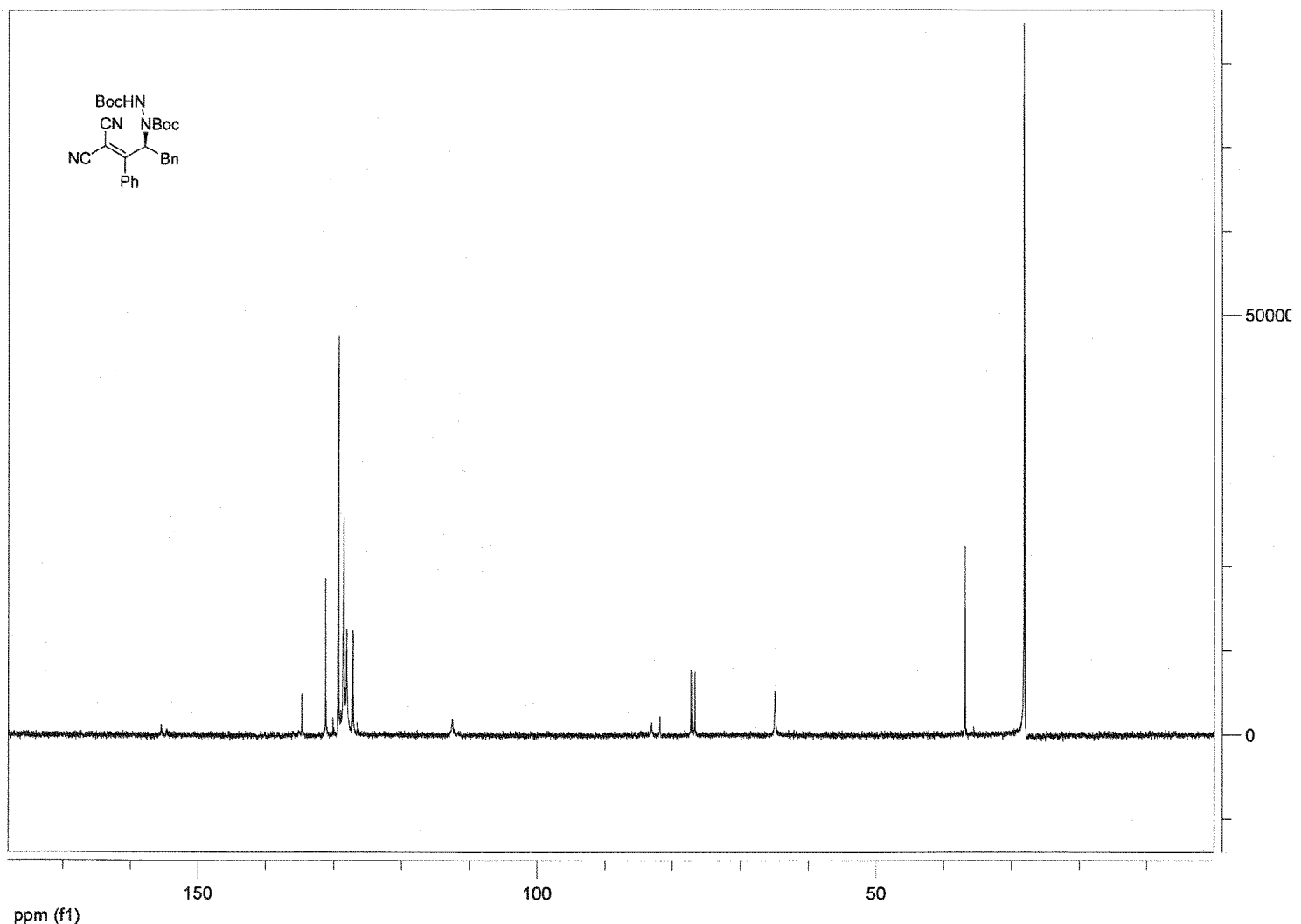




\section{(S)-(E)-4-[N,N'-Bis(tert-butoxycarbonyl)-hydrazino]-2-cyano-5-phenylpent-2-enoic}

acid benzyl ester (2o):

The title compound was obtained according to the general<smiles>CC(=C[C@@H](C#N)NC(=O)OCc1ccccc1)C(=O)O[Na]</smiles>
procedure after $\mathrm{FC}$ in $\mathrm{Et}_{2} \mathrm{O} / n$-hexane as a viscous colorless oil (85\% yield). ${ }^{1} \mathrm{H} \mathrm{NMR}\left(\mathrm{CDCl}_{3}, 60{ }^{\circ} \mathrm{C}\right) \delta 7.7(\mathrm{~d}, J 8.9 \mathrm{~Hz}, 1 \mathrm{H})$, 7.42-7.20 (m, 10H), 6.11 (br s, 1H), $5.36(\mathrm{~m}, 1 \mathrm{H}), 5.28(\mathrm{~s}, 2 \mathrm{H})$, $3.15(\mathrm{~m}, 1 \mathrm{H}), 2.94(\mathrm{dd}, J 13.4,6.2 \mathrm{~Hz}, 1 \mathrm{H}), 1.46(\mathrm{~s}, 9 \mathrm{H}), 1.40(\mathrm{~s}, 9 \mathrm{H}) \cdot{ }^{13} \mathrm{C} \mathrm{NMR}\left(\mathrm{CDCl}_{3}\right.$, $\left.60{ }^{\circ} \mathrm{C}\right) \delta 160.7,159.5,156.0,153.9,135.9,134.9,129.1$ (2C), 128.8 (2C), $128.6(2 \mathrm{C})$, 128.5 (2C), 128.2, 127.2, 112.8, 109.5, 82.5, 82.1, 68.0, 60.5, 37.7, 28.0 (6C). HRMS calc.: $\mathrm{C}_{29} \mathrm{H}_{35} \mathrm{~N}_{3} \mathrm{NaO}_{6}$ 524.2424; found: 524.2410. [ $\left.\alpha\right]_{\mathrm{D}}{ }^{20}+95\left(c=0.49, \mathrm{CH}_{2} \mathrm{Cl}_{2}, 98 \%\right.$ ee). The ee was determined by HPLC using a Chiralpak AD column [hexane/iPrOH (80:20)]; flow rate $1.0 \mathrm{~mL} / \mathrm{min} ; \tau_{\text {major }}=13.7 \mathrm{~min}, \tau_{\text {minor }}=26.7 \mathrm{~min}(98 \%$ ee $)$.

\section{Procedure for reduction of $2 \mathrm{i}$ to 3.}

To an oven-dried flask equipped with a magnetic stirring bar were added $2 \mathbf{i}(215 \mathrm{mg}$, $0.480 \mathrm{mmol}, 98 \%$ ee), $10 \% \mathrm{Pd} / \mathrm{C}(50 \mathrm{mg})$, and $\mathrm{EtOH}(5 \mathrm{~mL})$. The resulting mixture was cooled to $0{ }^{\circ} \mathrm{C}$ and stirred for 45 min under $\mathrm{H}_{2}(1 \mathrm{~atm})$. The mixture was filtered and the solvent was removed in vacuo. The pure product (193 mg, $0.432 \mathrm{mmol}, 90 \%, 1: 1.5$ mixture of diastereomers) was obtained after $\mathrm{FC}$ on $\mathrm{SiO}_{2}$ eluting with EtOAc/ $n$-hexane $(20: 80)$.

$(2 S, 4 S) \quad$ and $\quad(2 R, 4 S)-4-\left[N, N^{\prime}\right.$-Bis(tert-butoxycarbonyl)-hydrazino]-2-cyano-5phenylpentanoic acid methyl ester (3i):<smiles>CC(=O)OC(C)CC(Br)[N+](NC(=O)OCc1ccccc1)C(=O)O</smiles>

${ }^{1} \mathrm{H}$ NMR (benzene- $\left.\mathrm{d}_{6}, 60{ }^{\circ} \mathrm{C}\right) \delta$ 7.08-6.82 (m, 10H), 5.79 (br s, $2 \mathrm{H}), 4.75(\mathrm{~m}, 2 \mathrm{H}), 3.24(\mathrm{~s}, 3 \mathrm{H}), 3.22(\mathrm{~s}, 3 \mathrm{H}), 2.78-2.22(\mathrm{~m}$, $6 \mathrm{H}), 2.02(\mathrm{~m}, 2 \mathrm{H}), 1.75(\mathrm{~m}, 2 \mathrm{H}), 1.39(\mathrm{~s}, 9 \mathrm{H}), 1.36(\mathrm{~s}, 9 \mathrm{H})$, $1.33(\mathrm{~s}, 9 \mathrm{H}), 1.28(\mathrm{~s}, 9 \mathrm{H}) .{ }^{13} \mathrm{C}$ NMR (benzene-d $\left.6,60{ }^{\circ} \mathrm{C}\right)$ $\delta 166.9$ (2C), 156.7 (2C), 154.9 (2C), 138.1 (2C), 129.1 (4C), 128.9 (2C), 128.8 (2C), 126.9, 126.8, 117.1, 116.9, 81.6 (2C), 81.4 (2C), 57.8, 56.3, 52.7, 52.7, 39.1 (2C), 35.0, 33.9, 32.4 (2C), 28.2 (3C), 28.1 (3C), 28.0 (6C). HRMS calc.: $\mathrm{C}_{23} \mathrm{H}_{33} \mathrm{~N}_{3} \mathrm{NaO}_{6}$ 470.2267; Found: 470.2269. The ee was determined by HPLC using a Chiralcel OD column 
[hexane $/ \mathrm{iPrOH}(99: 1)]$; flow rate $1.0 \mathrm{~mL} / \mathrm{min} ; \tau_{\text {major }}=17.3 \mathrm{~min}, \tau_{\text {minor }}=19.9 \mathrm{~min}(98 \%$ ee). The stereocenter at $\mathrm{C} 2$ equilibrates so rapidly during the HPLC analysis that only one set of peaks is observed.

Procedure for Diels-Alder reaction of 2i with 2,3-dimethyl-1,3-butadiene. To a screw capped vial equipped with a magnetic stirrer bar were added $\mathbf{2 i}(106 \mathrm{mg}, 0.237 \mathrm{mmol}$, $98 \%$ ee) and 2,3-dimethyl-1,3-butadiene ( $288 \mu \mathrm{L}, 2.5 \mathrm{mmol})$ and $3.5 \mathrm{~mL}$ of toluene. The mixture was stirred at $80{ }^{\circ} \mathrm{C}$ for $23 \mathrm{~h}$ and was then cooled to room temperature, concentrated in vacuo and subjected to $\mathrm{FC}$ on $\mathrm{SiO}_{2}$ eluting with $\mathrm{Et}_{2} \mathrm{O}$ in $n$-hexane (10:90 to 20:80) to afford the Diels-Alder cycloadduct 4 as a white solid (108 $\mathrm{mg}, 0.204 \mathrm{mmol}$, $86 \%$ ). The compound was obtained as a $>15: 1$ ratio of diastereomers favoring the isomer shown below (the diastereomers can be separated under the chromatographic conditions mentioned above).

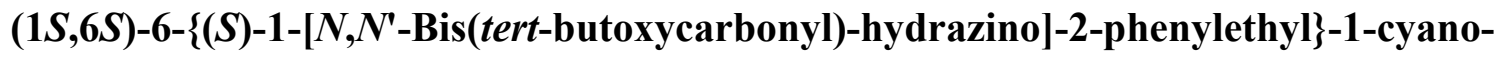
3,4-dimethylcyclohex-3-ene carboxylic acid methyl ester (4):

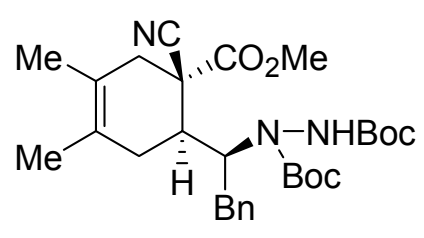

${ }^{1} \mathrm{H}$ NMR $\left(\mathrm{CDCl}_{3}, 60{ }^{\circ} \mathrm{C}\right) \delta$ 7.34-7.17 (m, 5H), 6.24 (br s, $\left.1 \mathrm{H}\right)$, $5.07(\mathrm{~m}, 1 \mathrm{H}), 3.84(\mathrm{~s}, 3 \mathrm{H}), 3.25(\mathrm{~m}, 1 \mathrm{H}), 2.86(\mathrm{~m}, 1 \mathrm{H}), 2.61-$ $2.31(\mathrm{~m}, 3 \mathrm{H}), 2.30-2.14(\mathrm{~m}, 2 \mathrm{H}), 1.68(\mathrm{~s}, 3 \mathrm{H}), 1.57(\mathrm{~s}, 3 \mathrm{H})$, $1.50(\mathrm{~s}, 9 \mathrm{H}), 1.48(\mathrm{~s}, 9 \mathrm{H}) \cdot{ }^{13} \mathrm{C} \mathrm{NMR}\left(\mathrm{CHCl}_{3}, 60{ }^{\circ} \mathrm{C}\right)^{5} \delta 169.2$, $156.0,155.1,137.9,128.9$ (2C), 128.6 (2C), 126.4, 126.3, $120.3,119.2,81.5,81.1,54.4,53.4,53.3,46.8,42.4,40.7,35.2,29.1,28.2$ (3C), 28.1

(3C), 18.9, 18.1. HRMS calc.: $\mathrm{C}_{29} \mathrm{H}_{41} \mathrm{~N}_{3} \mathrm{NaO}_{6} 550.2893$; Found: 550.2892. $[\alpha]_{\mathrm{D}}{ }^{20}+17(c$ $=0.55, \mathrm{CHCl}_{3}, 98 \%$ ee). The ee was determined by HPLC using a Chiralpak AD column [hexane $/ \mathrm{iPrOH}(97: 3)$ ]; flow rate $1.0 \mathrm{~mL} / \mathrm{min} ; \tau_{\text {minor }}=6.2 \mathrm{~min}, \tau_{\text {major }}=6.9 \mathrm{~min}(98 \%$ ee). 
The relative configuration of the Diels-Alder cycloadduct was determined by X-ray crystallography (Figure 1).
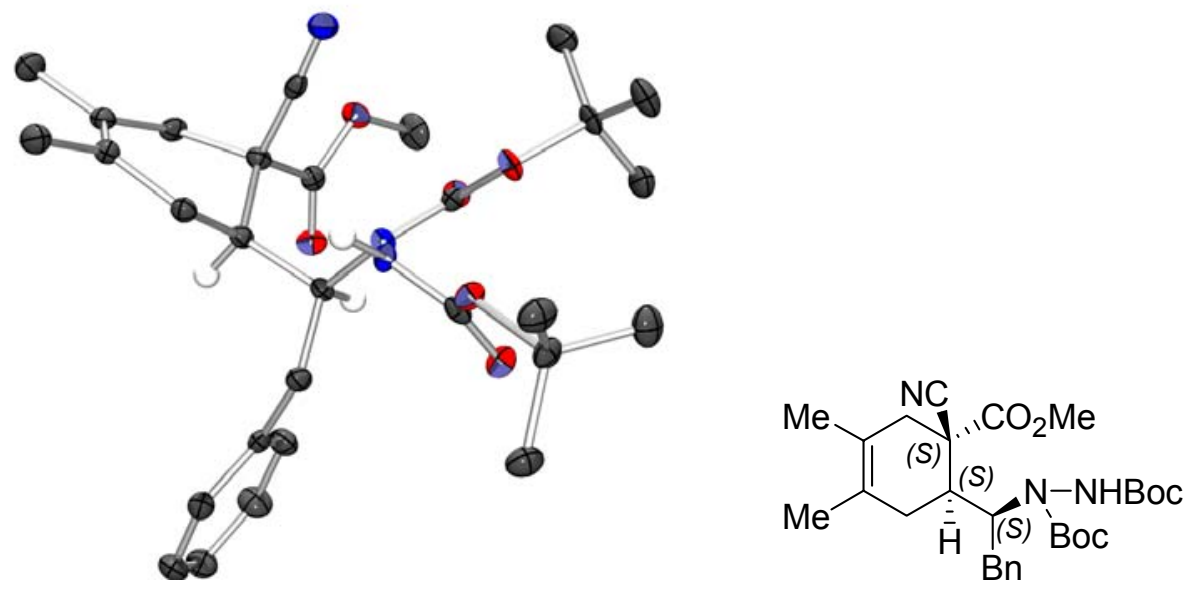

Figure 1. X-ray crystal structure of Diels-Alder cycloadduct (4). Most hydrogens have been omitted for clarity.

\section{Procedure for reduction and decarboxylation of 20.}

A flask equipped with a magnetic stirring bar was loaded with 20 (418 $\mathrm{mg}, 0.801 \mathrm{mmol}$, $98 \%$ ee). Then $\mathrm{EtOH}(10 \mathrm{~mL})$ and $10 \% \mathrm{Pd} / \mathrm{C}(80 \mathrm{mg})$ were added. The resulting mixture was stirred at ambient temperature under $\mathrm{H}_{2}(1 \mathrm{~atm})$. After $2 \mathrm{~h}$ the mixture was filtered and the solvent was removed in vacuo. Then DMF $(4 \mathrm{~mL})$ was added and the solution was placed in an oil bath (preheated to $150{ }^{\circ} \mathrm{C}$ ). After stirring at $150{ }^{\circ} \mathrm{C}$ for $2 \mathrm{~h}$ the solution was cooled to room temperature and $\mathrm{H}_{2} \mathrm{O}(10 \mathrm{~mL})$ was added. The mixture was extracted with $\mathrm{Et}_{2} \mathrm{O}(4 \times 25 \mathrm{~mL})$ and the combined organic extracts were washed with brine $(10 \mathrm{~mL})$ and dried over $\mathrm{MgSO}_{4}$. After removal of the drying agent, the solvent was evaporated and the pure product 5 ( $237 \mathrm{mg}, 0.609 \mathrm{mmol}, 76 \%$ yield) was isolated by FC on $\mathrm{SiO}_{2}$ eluting with $\mathrm{Et}_{2} \mathrm{O}$ in $\mathrm{CH}_{2} \mathrm{Cl}_{2}(0: 100$ to $15: 85)$ as a colorless viscous oil. 


\section{(R)-4-(N,N'-Bis(tert-butoxycarbonyl-hydrazino)-5-phenylpentannitrile}

${ }^{1} \mathrm{H}$ NMR $\left(\mathrm{CDCl}_{3}, 60{ }^{\circ} \mathrm{C}\right) \oint 7.34-7.14(\mathrm{~m}, 5 \mathrm{H}), 5.78$ (br s, $\left.1 \mathrm{H}\right), 4.52(\mathrm{~m}$,<smiles>CCCC[C@H](Br)C(=O)N[Nb]C(=O)OCc1ccccc1</smiles>

1H), 2.95-2.60 (m, 4H), $1.93(\mathrm{~m}, 1 \mathrm{H}), 1.78(\mathrm{~m}, 1 \mathrm{H}), 1.50(\mathrm{~s}, 9 \mathrm{H}), 1.39$

$(\mathrm{s}, 9 \mathrm{H}) .{ }^{13} \mathrm{C} \mathrm{NMR}\left(\mathrm{CDCl}_{3}, 60{ }^{\circ} \mathrm{C}\right) \delta 155.0,154.3,137.8,128.7$ (4C), 126.7, 119.9, 81.7 (2C), 58.6, 38.9, 28.4, 28.2 (3C), 28.1 (3C), 14.6.

HRMS calc.: $\mathrm{C}_{21} \mathrm{H}_{31} \mathrm{~N}_{3} \mathrm{NaO}_{4}$ 412.2212; found: 412.2224. $[\alpha]_{\mathrm{D}}{ }^{20}+14$ (c $=1.0, \mathrm{CHCl}_{3}$, $98 \%$ ee). The ee was determined by HPLC using a Chiralcel AD column [hexane $/ \mathrm{PrOH}$ (90:10)]; flow rate $1.0 \mathrm{~mL} / \mathrm{min} ; \tau_{\text {major }}=8.6 \mathrm{~min}, \tau_{\text {minor }}=11.7 \mathrm{~min}(98 \%$ ee $)$.

\section{Procedure for $\mathrm{N}-\mathrm{N}$ bond cleavage of 5.}

Compound 5 (237 mg, $0.609 \mathrm{mmol}$ ) was placed in an oven-dried flask equipped with a magnetic stirring bar. $\mathrm{Ac}_{2} \mathrm{O}(980 \mu \mathrm{L}, 10.4 \mathrm{mmol})$, pyridine $(510 \mu \mathrm{L}, 6.1 \mathrm{mmol})$, and DMAP (10 mol\%, $0.061 \mathrm{mmol}, 9 \mathrm{mg}$ ) were added and the mixture was stirred under an argon atmosphere at $50{ }^{\circ} \mathrm{C}$ for $49 \mathrm{~h}$. The mixture was then cooled to room temperature and diluted with $\mathrm{Et}_{2} \mathrm{O}$ (ca. $7 \mathrm{~mL}$ ). To the diluted mixture was added $1 \mathrm{M} \mathrm{NaHCO}_{3}$ (aq.) $(10 \mathrm{~mL})$ and stirring was continued at ambient temperature for $30 \mathrm{~min}$. The layers were then separated and the aqueous layer was extracted with $\mathrm{Et}_{2} \mathrm{O}(2 \times 15 \mathrm{~mL})$ and EtOAc (1 x $15 \mathrm{~mL}$ ). The combined organic extracts were washed successively with $1 \mathrm{M} \mathrm{NaHCO}_{3}$ (aq.) $(10 \mathrm{~mL}), 1 \mathrm{M} \mathrm{HCl}$ (aq.) $(10 \mathrm{~mL})$, and brine $(10 \mathrm{~mL})$. The solution was dried over $\mathrm{Na}_{2} \mathrm{SO}_{4}$ and the solvent was evaporated. $\mathrm{FC}$ on $\mathrm{SiO}_{2}$ eluting with $\mathrm{Et}_{2} \mathrm{O}$ in $\mathrm{CH}_{2} \mathrm{Cl}_{2}(0: 100$ to 5:95) afforded the $N$-acetylated product (192 $\mathrm{mg}, 0.445 \mathrm{mmol}, 73 \%$ ) and recovered starting material (47 mg, $0.122 \mathrm{mmol}, 20 \%$ ).

The acetylated product $(71.2 \mathrm{mg}, 0.165 \mathrm{mmol})$ was dissolved in dry and deoxygenated THF (2 mL) under an Ar-atmosphere. Deoxygenated HMPA (0.3 mL) was added, followed by addition of $8 \mathrm{~mL}$ of $\mathrm{SmI}_{2}$-solution (0.1M in THF) dropwise at room temperature. The resulting purple solution was stirred for $30 \mathrm{~min}$ at room temperature and was then quenced by addition of $5 \mathrm{~mL} 1 \mathrm{M} \mathrm{NaHCO}_{3}$ (aq). The solution was diluted with EtOAc and placed in a separating funnel. The layers were separated and the aqueous phase was extracted with EtOAc $(2 \times 20 \mathrm{~mL})$. The combined organic extracts were washed with brine and dried $\left(\mathrm{MgSO}_{4}\right)$ and concentrated in vacuo. The pure product $(42.3$ 
mg, 0.154 mol, 93\%) was obtained as an off-white solid $\left(\mathrm{mp}=93-95{ }^{\circ} \mathrm{C}\right)$ by $\mathrm{FC}$ on $\mathrm{SiO}_{2}$ eluting with $\mathrm{Et}_{2} \mathrm{O}$ in $\mathrm{CH}_{2} \mathrm{Cl}_{2}$ (0:100 to 10:90).

\section{(R)-(1-Benzyl-3-cyano-propyl)-carbamic acid tert-butyl ester}

${ }^{1} \mathrm{H}$ NMR $\left(\mathrm{CDCl}_{3}\right) \delta 7.31(\mathrm{~m}, 2 \mathrm{H}), 7.25(\mathrm{~m}, 1 \mathrm{H}), 7.16(\mathrm{~m}, 2 \mathrm{H}), 4.37$ (br<smiles>CC(C)(C)OC(=O)N[C@@H](N)CCC#N</smiles>
d, J 9.2 Hz, 1H), 3.85 (m, 1H), 2.87 (dd, J, 6.0, $13.7 \mathrm{~Hz}, 1 \mathrm{H}), 2.76$ (dd, $J$ 6.9, $13.4 \mathrm{~Hz}, 1 \mathrm{H}), 2.47-2.31(\mathrm{~m}, 2 \mathrm{H}), 1.92(\mathrm{~m} 1 \mathrm{H}), 1.70(\mathrm{~m}, 1 \mathrm{H})$, $1.41(\mathrm{~s}, 9 \mathrm{H}) .{ }^{13} \mathrm{C} \mathrm{NMR}\left(\mathrm{CDCl}_{3}\right) \delta 155.4,137.0,129.3(2 \mathrm{C}), 128.6$ (2C), 126.8, 119.6, 79.9, 51.2, 41.3, 30.4, 28.3 (3C), 14.4. HRMS calc.: $\mathrm{C}_{16} \mathrm{H}_{22} \mathrm{~N}_{2} \mathrm{NaO}_{2}$ 297.1579; found: $297.1578 .[\alpha]_{\mathrm{D}}{ }^{20}+13\left(\mathrm{c}=1.0, \mathrm{CHCl}_{3}, 98 \%\right.$ ee). The ee was determined by HPLC using a Chiralcel OJ column [hexane/ $\mathrm{PrOH}(90: 10)]$; flow rate $1.0 \mathrm{~mL} / \mathrm{min}$; $\tau_{\text {major }}=14.2 \mathrm{~min}, \tau_{\text {minor }}=11.9 \min (98 \%$ ee $)$

\section{Determination of the Absolute Configuration of the Allylic Amination Products:}

The absolute configuration was established by correlation with $\alpha$-hydrazino aldehydes obtained by L-proline catalyzed $\alpha$-amination. ${ }^{4}$ When using (DHQ) ${ }_{2} \mathrm{PYR}$ as the catalyst the configuration of the products was found to be $(S)$.
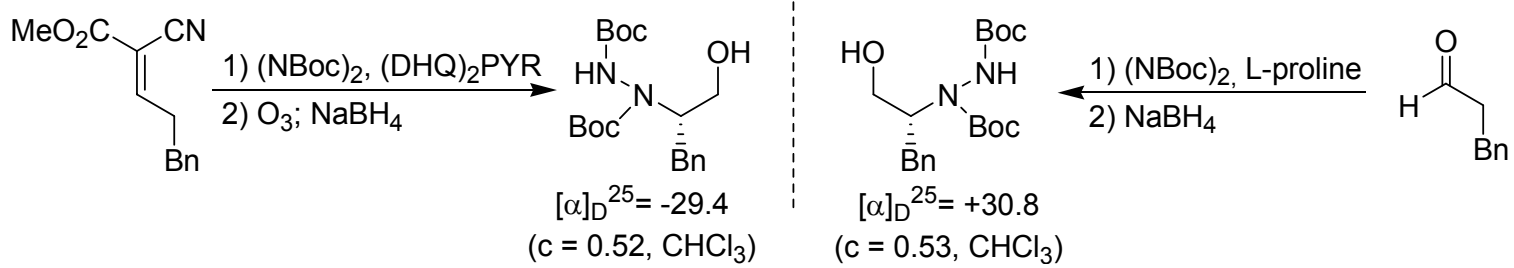


\section{References}

1. Belle, D. D.; Tolvanen, A.; Lounasmaa, M. Tetrahedron, 1996, 52, 11361.

2. Hoffman, J. M.; Smith, A. M.; Rooney, C. S.; Fisher, T. E.; Wai, J. S.; Thomas, C. M.; Bamberger, D. L.; Barnes, J. L.; Williams, T. M.; Jones, J. H.; Olson, B. D.; O’Brien, J. A.; Goldman, M. E.; Nunberg, J. H.; Quintero, J. C.; Schleif, W. A.; Emini, E. A.; Anderson, P. S. J. Med. Chem. 1993, 36, 953.

3. Sammelson, R. E.; Allen, M. J.; Synthesis, 2005, 543.

4. (a) Bøgevig, A.; Juhl, K.; Kumaragurubaran, N.; Zhuang, W.; Jørgensen, K. A. Angew. Chem. Int. Ed. 2002, 41, 1790; (b) List, B. J. Am. Chem. Soc. 2002, 124, 5656.

5. Due to the presence of distinct rotameric isomers, the ${ }^{13} \mathrm{C}$ NMR spectrum contain extra peaks. 